\title{
APPROXIMATION PROPERTIES OF THE PICARD SINGULAR INTEGRAL IN EXPONENTIAL WEIGHTED SPACES
}

\author{
A. Leśniewicz, L. Rempulska And J. WAsiak
}

Abstract

In this note we give some direct and inverse approximation theorems for the Picard singular integral in the exponential weighted spaces and some generalized Hölder spaces.

\section{Preliminaries}

1.1. The Picard singular integral

$$
P_{r}(f ; x):=\frac{1}{2 r} \int_{-\infty}^{+\infty} f(x+t) \exp \left(-\frac{|t|}{r}\right) d t
$$

$x \in \mathbb{R}:=(-\infty,+\infty), r>0$ and $r \rightarrow 0_{+}$, was examined in $[\mathbf{1}],[\mathbf{2}],[\mathbf{4}]$ for functions belonging to the space $L^{p}$ and the classical Hölder spaces.

The purpose of this note is to give some approximation properties of the Picard integral (1) in the exponential weighted spaces $L^{p, q}$ and some generalized Hölder spaces [5].

1.2. Let $q>0$ be a fixed number and let

$$
w_{q}(x):=e^{-q|x|}, \quad x \in \mathbb{R} .
$$

For a fixed $1 \leq p \leq \infty$ and $q>0$ we denote by $L^{p, q}$ the set of all real-valued functions $f$ defined on $\mathbb{R}$ for which the $p$-th power of $w_{q} f$ is Lebesgue-integrable on $\mathbb{R}$ if $1 \leq p<\infty$, and $w_{q} f$ is uniformly continuous and bounded on $\mathbb{R}$ if $p=\infty$. Let the norm in $L^{p, q}$ be given by the formula

(3) $\quad\|f\|_{p, q} \equiv\|f(\cdot)\|_{p, q}:= \begin{cases}\left(\int_{\mathbb{R}}\left|w_{q}(x) f(x)\right|^{p} d x\right)^{\frac{1}{p}} & \text { if } 1 \leq p<\infty \\ \sup _{x \in \mathbb{R}} w_{q}(x)|f(x)| & \text { if } p=\infty\end{cases}$ 
For $f \in L^{p, q}$ we define the modulus of smoothness of the order 2

$$
\omega_{2}\left(f, L^{p, q} ; t\right):=\sup _{|h| \leq t}\left\|\Delta_{h}^{2} f(\cdot)\right\|_{p, q}, \quad t \geq 0
$$

where

$$
\Delta_{h}^{2} f(x):=f(x+h)+f(x-h)-2 f(x), \quad x, h \in \mathbb{R} .
$$

From (3)-(5) for $f \in L^{p, q}$ follows

$$
\|f(\cdot+h)\|_{p, q} \leq e^{q|h|}\|f(\cdot)\|_{p, q}, \quad h \in \mathbb{R},
$$

(7) $0=\omega_{2}\left(f, L^{p, q} ; 0\right) \leq \omega_{2}\left(f, L^{p, q} ; t_{1}\right) \leq \omega_{2}\left(f, L^{p, q} ; t_{2}\right)$ if $0<t_{1}<t_{2}$.

Using the indentity (see [3, pp. 25-29])

$$
\Delta_{n h}^{2} f(x)=\sum_{k=1}^{n} k \Delta_{h}^{2} f(x-(n-k) h)+\sum_{k=1}^{n-1}(n-k) \Delta_{h}^{2} f(x+k h),
$$

$x, h \in \mathbb{R} ; n=2,3, \ldots$, and by (2)-(6) we can prove that

$$
\omega_{2}\left(f, L^{p, q} ; n t\right) \leq n^{2} e^{(n-1) q t} \omega_{2}\left(f, L^{p, q} ; t\right) \text { for } n=1,2, \ldots \text { and } t \geq 0
$$

and

$$
\omega_{2}\left(f, L^{p, q} ; \lambda t\right) \leq(\lambda+1)^{2} e^{\lambda q t} \omega_{2}\left(f, L^{p, q} ; t\right) \text { for } \lambda, t \geq 0
$$

1.3. Denote as in [5] by $\Omega^{2}$ the set of all functions of order 2 modulus of smoothness type $([\mathbf{6}])$, i.e., $\Omega^{2}$ is the set of all functions $\omega$ satisfying the following conditions

(i) $\omega$ is defined and continuous on $[0,+\infty)$,

(ii) $\omega$ is increasing and $\omega(0)=0$,

(iii) $\omega(t) t^{-2}$ is decreasing on $[0,+\infty)$.

As in [5], for a given $1 \leq p \leq \infty, q>0$ and $\omega \in \Omega^{2}$, we define the generalized Hölder space $L^{p}, q, \omega$ of all functions $f \in L^{p, q}$ for which the quantity

$$
\|f\|_{p, q, \omega}^{*}:=\sup _{0<h \leq 1}\left\{\frac{\left\|\Delta_{h}^{2} f(\cdot)\right\|_{p, q}}{\omega(h)}\right\}
$$

is finite. The norm in $L^{p, q, \omega}$ is defined by

$$
\|f\|_{p, q, \omega}:=\|f\|_{p, q}+\|f\|_{p, q, \omega}^{*}
$$


For $f \in L^{p, q, \omega}$ we have

$$
\omega_{2}\left(f, L^{p, q} ; t\right) \leq\|f\|_{p, q, \omega}^{*} \omega(t), \quad 0 \leq t \leq 1 .
$$

If $\omega, \mu \in \Omega^{2}$ and the function

$$
\varphi(t):=\frac{\omega(t)}{\mu(t)}, \quad t>0,
$$

is increasing, then for a fixed $1 \leq p \leq \infty$ and $q>0$ we have $L^{p, q, \omega} \subset$ $L^{p, q, \mu}$.

It is easy to observe that for every $\omega \in \Omega^{2}$ there exist two positive constants $M_{1}, M_{2}$ such that

$$
M_{1} t^{2} \leq \omega(t) \leq M_{2} t^{2} \int_{t}^{1} \omega(z) z^{-3} d z \text { for all } 0 \leq t \leq \frac{1}{2}
$$

In this note we shall study the limit properties of the Picard integral (1) for functions belonging to the spaces $L^{p, q}$ and $L^{p, q, \omega}$. We first notice that for each $r, P_{r}$, as given by (1), is well defined on all functions $f \in L^{p, q}$, $1 \leq p \leq \infty, q>0$, provided $r$ is small enough, i.e., $0<r<\frac{1}{q}$. It is then a linear positive operator. In Section 2 we shall prove that, for a given $1 \leq p \leq \infty, q>0$ and $0<r<\frac{1}{q}, P_{r}$ is an operator from $L^{p, q}$ into $L^{p, q}$. Moreover, we shall prove that, for a given $1 \leq p \leq \infty, q>0, \omega \in \Omega^{2}$ and $0<r<\frac{1}{q}, P_{r}$ is an operator from $L^{p, q, \omega}$ into $L^{p, q, \omega}$.

\section{Auxiliary results}

In this part we shall give some fundamental properties of the Picard integral $P_{r}$ in the spaces $L^{p, q}$ and $L^{p, q, \omega}$.

It is easy to verify that holds.

Lemma 1. For $k=0,1,2, \ldots$ and $y>0$ we have

$$
\int_{0}^{+\infty} t^{k} \exp (-y t) d t=k ! y^{-k-1}
$$

In what follows, for a given $q>0$, we shall denote by $r_{0} \equiv r_{0}(q)$ a fixed number such that

$$
0<r_{0}<\frac{1}{q}
$$


Lemma 2. For every fixed $1 \leq p \leq \infty$ and $q>0$, the Picard integral $P_{r}$ is an operator from $L^{p, q}$ into $L^{p, q}$ provided that $0<r<\frac{1}{q}$. Moreover,

$$
\left\|P_{r}(f ; \cdot)\right\|_{p, q} \leq \frac{1}{1-r_{0} q}\|f\|_{p, q} \text { for } 0<r \leq r_{0},
$$

where $r_{0}$ is given by (14).

Proof: We shall prove only (15).

If $f \in L^{\infty, q}, q>0$, then by (1)-(3) we have

$$
\begin{aligned}
w_{q}(x)\left|P_{r}(f ; x)\right| & \leq\|f\|_{\infty, q} e^{-q|x|}(2 r)^{-1} \int_{\mathbb{R}} e^{q|x+t|-\frac{|t|}{r}} d t \\
& \leq\|f\|_{\infty, q} r^{-1} \int_{0}^{+\infty} e^{t\left(q-\frac{1}{r}\right)} d t, \quad x \in \mathbb{R},
\end{aligned}
$$

and further for $0<r \leq r_{0}<\frac{1}{q}$ we get by Lemma 1

$$
\left\|P_{r}(f ; \cdot)\right\|_{\infty, q} \leq\|f\|_{\infty, q} \frac{1}{1-r q} \leq \frac{1}{1-r_{0} q}\|f\|_{\infty, q} .
$$

Similarly, if $f \in L^{p, q}, 1 \leq p<\infty$ and $q>0$, then by (1)-(3) and the generalized Minkowski inequality we get

$$
\left\|P_{r}(f ; \cdot)\right\|_{p, q} \leq\|f\|_{p, q}(2 r)^{-1} \int_{\mathbb{R}} e^{|t|\left(q-\frac{1}{r}\right)} d t
$$

which by Lemma 1 implies (15) for $r \in\left(0, r_{0}\right]$. Thus the proof of (15) is completed.

Lemma 3. Let $L^{p, q, \omega}$ be a given Hölder space $(1 \leq p \leq \infty, q>0$, $\left.\omega \in \Omega^{2}\right)$ and let $r_{0}$ be given by (14). Then for every $f \in L^{p, q, \omega}$ and $r \in\left(0, r_{0}\right]$ holds

$$
\left\|P_{r}(f ; \cdot)\right\|_{p, q, \omega} \leq \frac{1}{1-r_{0} q}\|f\|_{p, q, \omega},
$$

which proves that $P_{r}, 0<r<\frac{1}{q}$, is an operator from $L^{p, q, \omega}$ into $L^{p, q, \omega}$.

Proof: From (1) and (5) follows

$$
\Delta_{h}^{2} P_{r}(f ; x)=P_{r}\left(\Delta_{h}^{2} f ; x\right)
$$

for all $x, h \in \mathbb{R}$ and $0<r<\frac{1}{q}$. Hence and by (9) and (15) we have

(18) $\left\|P_{r}(f ; \cdot)\right\|_{p, q, \omega}^{*}:=\sup _{0<h \leq 1}\left\{\frac{\left\|P_{r}\left(\Delta_{h}^{2} f ; \cdot\right)\right\|_{p, q}}{\omega(h)}\right\}$

$$
\leq \frac{1}{1-r_{0} q} \sup _{0<h \leq 1}\left\{\frac{\left\|\Delta_{h}^{2} f ;(\cdot)\right\|_{p, q}}{\omega(h)}\right\}=\frac{1}{1-r_{0} q}\|f\|_{p, q, \omega}^{*}
$$

for all $0<r \leq r_{0}<\frac{1}{q}$. Combining (15), (18) and (10), we obtain (16) and we complete the proof. 
Lemma 4. Suppose that $f \in L^{p, q}$ with some $1 \leq p \leq \infty, q>0$, and $r_{0}$ is given by (14). Then

(19) $\quad P_{r_{1}}\left(P_{r_{2}}(f) ; x\right)=P_{r_{2}}\left(P_{r_{1}}(f) ; x\right)$ for $x \in \mathbb{R}, \quad r_{1}, r_{2} \in\left(0, \frac{1}{q}\right)$.

Moreover, for every fixed $r \in\left(0, \frac{1}{q}\right)$ the function $P_{r}(f ; \cdot)$ has derivatives of all orders belonging also to $L^{p, q}$ and

$$
\left\|P_{r}^{(n)}(f ; \cdot)\right\|_{p, q} \leq\left(1-r_{0} q\right)^{-1} r^{-n}\|f\|_{p, q}
$$

for all $n=1,2, \ldots$ and $r \in\left(0, r_{0}\right]$.

Proof: For $f \in L^{p, q}, n=1,2, \ldots$ and $0<r<\frac{1}{q}$ we get from (1)

$$
P_{r}^{(n)}(f ; x)=r^{-n} P_{r}(f ; x), \quad x \in \mathbb{R} .
$$

This fact and Lemma 2 imply (20). The equality (19) we immediately obtain from (1) and Lemma 2 .

Lemma 5. Suppose that the assumption of Lemma 4 is satisfied. Then

$$
\left\|\Delta_{h}^{2} P_{r}(f ; \cdot)\right\|_{p, q} \leq \frac{1}{1-r_{0} q}\|f\|_{p, q} r^{-2} h^{2} e^{q|h|}
$$

for all $r \in\left(0, r_{0}\right]$ and $h \in \mathbb{R}$.

Proof: By Lemma 4 and (5) for $x, h \in \mathbb{R}$ and $0<r<\frac{1}{q}$ we can write

$$
\Delta_{h}^{2} P_{r}(f ; x)=\int_{-\frac{h}{2}}^{\frac{h}{2}} \int_{-\frac{h}{2}}^{\frac{h}{2}} P_{r}^{\prime \prime}\left(f ; x+t_{1}+t_{2}\right) d t_{1} d t_{2} .
$$

Now arguing as in the proof of Lemma 2 we get

$$
\begin{aligned}
\left\|\Delta_{h}^{2} P_{r}(f ; x)\right\|_{p, q}=\left\|P_{r}^{\prime \prime}(f ; \cdot)\right\|_{p, q} \int_{-\frac{h}{2}}^{\frac{h}{2}} \int_{-\frac{h}{2}}^{\frac{h}{2}} e^{q\left|t_{1}+t_{2}\right|} d t_{1} d t_{2} \\
\leq\left\|P_{r}^{\prime \prime}(f ; \cdot)\right\|_{p, q} h^{2} e^{q|h|}
\end{aligned}
$$

which by (20) yields the desired inequality (21). 


\section{Approximation theorems}

3.1. First we shall prove a direct approximation theorem for functions belonging to $L^{p, q}$.

Theorem 1. If $f \in L^{p, q}$ with some $1 \leq p \leq \infty$ and $q>0$, and if $r_{0}$ is given by (14), then

$$
\left\|P_{r}(f ; \cdot)-f(\cdot)\right\|_{p, q} \leq \frac{5}{2}\left(1-r_{0} q\right)^{-3} \omega_{2}\left(f, L^{p, q} ; r\right)
$$

for all $r \in\left(0, r_{0}\right]$.

Proof: From (1), Lemma 1 and (5) follows

$$
P_{r}(f ; x)-f(x)=\frac{1}{2 r} \int_{0}^{+\infty}\left(\Delta_{t}^{2} f(x)\right) e^{-\frac{t}{r}} d t
$$

for $x \in \mathbb{R}$ and $r \in\left(0, \frac{1}{q}\right)$. Further, by some calculations as in the proof of Lemma 2 and by (4) and (8), we get

$$
\begin{aligned}
\left\|P_{r}(f)-f\right\|_{p, q} & \leq \frac{1}{2 r} \int_{0}^{+\infty}\left\|\Delta_{t}^{2} f(\cdot)\right\|_{p, q} e^{-\frac{t}{r}} d t \leq \frac{1}{2 r} \int_{0}^{+\infty} \omega_{2}\left(f, L^{p, q} ; t\right) e^{-\frac{t}{r}} d t \\
& \leq \omega_{2}\left(f, L^{p, q} ; r\right) \frac{1}{2 r} \int_{0}^{+\infty}\left(\frac{t}{r}+1\right)^{2} e^{-\left(\frac{1}{r}-q\right) t} d t .
\end{aligned}
$$

Now using Lemma 1, we easily obtain (22).

Theorem 1 and (11) imply the following

Corollary 1. If $f \in L^{p, q, \omega}$ with some fixed $1 \leq p \leq \infty, q>0$ and $\omega \in \Omega^{2}$, then

$$
\left\|P_{r}(f ; \cdot)-f(\cdot)\right\|_{p, q} \leq \frac{5}{2}\left(1-r_{0} q\right)^{-3}\|f\|_{p, q, \omega}^{*} \omega(r)
$$

for all $r \in(0,1] \cap\left(0, r_{0}\right]$, where $0<r_{0}<\frac{1}{q}$.

In particular, if $\omega(t)=t^{\alpha}$ with some $0<\alpha \leq 2$, then

$$
\left\|P_{r}(f ; \cdot)-f(\cdot)\right\|_{p, q}=O\left(r^{\alpha}\right) \text { as } r \rightarrow 0_{+} .
$$

3.2. Using the Bernstein method ([6, p. 345]) we shall prove an inverse approximation theorem for the Picard integral $P_{r}$. 
Theorem 2. Suppose that $f \in L^{p, q}$ with some $1 \leq p \leq \infty, q>0$ and

$$
\left\|P_{r}(f ; \cdot)-f(\cdot)\right\|_{p, q} \leq \omega(r) \text { for }\left(0, r_{0}\right]
$$

where $\omega$ is a given function belonging to $\Omega^{2}$ and $0<r_{0}<\frac{1}{q}$. Then there exists a positive constant $M$ depending only on $p, q, \omega, r_{0}$ and $\|f\|_{p, q}$ such that

$$
\omega_{2}\left(f, L^{p, q} ; t\right) \leq M t^{2} \int_{t}^{1} \omega(z) z^{-3} d z
$$

for all $t \in\left(0, \frac{1}{2}\right) \cap\left(0, \frac{1}{q}\right)$.

Proof: Choosing two natural numbers $n_{0}$ and $n$ such that $0<2^{-n}<$ $2^{-n_{0}}<\frac{1}{q}$, we can write

$$
\begin{aligned}
f(x)=P_{2^{-n_{0}}}(f ; x)+\sum_{k=n_{0}}^{n-1}\left\{P_{2^{-k-1}}(f ; x)\right. & \left.-P_{2^{-k}}(f ; x)\right\} \\
& +f(x)-P_{2^{-n}}(f ; x), \quad x \in \mathbb{R},
\end{aligned}
$$

and further by (5)

$$
\begin{aligned}
\Delta_{h}^{2} f(x)=\Delta_{h}^{2} P_{2^{-n_{0}}}(f ; x) & +\sum_{k=n_{0}}^{n-1} \Delta_{h}^{2}\left\{P_{2^{-k-1}}(f ; x)-P_{2^{-k}}(f ; x)\right\} \\
& +\Delta_{h}^{2}\left\{f(x)-P_{2-n}(f ; x)\right\}, \quad x, h \in \mathbb{R} .
\end{aligned}
$$

Using Lemma 5, we get

$$
\left\|\Delta_{h}^{2} P_{2^{-n_{0}}}(f ; \cdot)\right\|_{p, q} \leq\left(1-r_{0} q\right)^{-1}\|f\|_{p, q} 2^{2 n_{0}} h^{2} e^{q|h|} .
$$

From (1), (5) and (19) follows

$$
\begin{aligned}
& \Delta_{h}^{2}\left\{P_{2^{-k-1}}(f ; x)-P_{2^{-k}}(f ; x)\right\} \\
& \quad=\Delta_{h}^{2} P_{2^{-k-1}}\left(f-P_{2^{-k}}(f) ; x\right)+\Delta_{h}^{2} P_{2^{-k}}\left(P_{2^{-k-1}}(f)-f ; x\right) .
\end{aligned}
$$

Hence using Lemma 5 and (23), we obtain

$$
\begin{aligned}
& \left\|\Delta_{h}^{2}\left\{P_{2^{-k-1}}(f ; \cdot)-P_{2^{-k}}(f ; \cdot)\right\}\right\|_{p, q} \\
& \leq\left\|\Delta_{h}^{2} P_{2^{-k-1}}\left(f-P_{2^{-k}}(f) ; \cdot\right)\right\|_{p, q}+\left\|\Delta_{h}^{2} P_{2^{-k}}\left(f-P_{2^{-k-1}}(f) ; \cdot\right)\right\|_{p, q} \\
& \leq\left(1-r_{0} q\right)^{-1} e^{q|h|} h^{2}\left\{2^{2 k+2}\left\|f-P_{2^{-k}}(f)\right\|_{p, q}+2^{2 k}\left\|f-P_{2-k-1}(f)\right\|_{p, q}\right\} \\
& \leq\left(1-r_{0} q\right)^{-1} e^{q|h|} h^{2} 2^{2 k+2} \omega\left(2^{-k}\right) \text { for } h \in \mathbb{R} .
\end{aligned}
$$


By (5), (6) and (23) we have for $h \in \mathbb{R}$

$$
\begin{aligned}
& \left\|\Delta_{h}^{2}\left\{f(\cdot)-P_{2^{-n}}(f ; \cdot)\right\}\right\|_{p, q} \\
& \leq\left\|f(\cdot+h)-P_{2^{-n}}(f ; \cdot+h)\right\|_{p, q}+\left\|f(\cdot-h)-P_{2^{-n}}(f ; \cdot-h)\right\|_{p, q} \\
& +2\left\|f(\cdot)-P_{2^{-n}}(f ; \cdot)\right\|_{p, q} \leq 2\left(e^{q|h|}+1\right) \omega\left(2^{-n}\right) .
\end{aligned}
$$

Consequently

$$
\begin{aligned}
\left\|\Delta_{h}^{2} f(\cdot)\right\|_{p, q} \leq e^{q|h|}\left\{4^{n_{0}}\left(1-r_{0} q\right)^{-1} h^{2}\|f\|_{p, q}\right. \\
\left.+\left(1-r_{0} q\right)^{-1} h^{2} \sum_{k=n_{0}}^{n-1} 2^{2 k+3} \omega\left(2^{-k}\right)+4 \omega\left(2^{-n}\right)\right\}
\end{aligned}
$$

for all $h \in \mathbb{R}$. Now let $t \in\left(0, \frac{1}{2}\right) \cap\left(0, \frac{1}{q}\right),|h| \leq t, n_{0}<n$ and let $n$ be a natural number such that $2^{-n} \leq t<2^{-n+1}$. Then we get form (25)

$$
\omega_{2}\left(f, L^{p, q} ; t\right) \leq M_{1}\left\{t^{2}+t^{2} \sum_{k=n_{0}}^{n-1} 2^{2 k+3} \omega\left(2^{-k}\right)+\omega(t)\right\}
$$

where $M_{1}=e\left\{4^{n_{0}}\left(1-r_{0} q\right)^{-1}\|f\|_{p, q}+\left(1-r_{0} q\right)^{-1}+4\right\}$. Since $\omega(s) s^{-2}$ is decreasing for $s>0$, we obtain

$$
\sum_{k=n_{0}}^{n-1} 2^{2 k} \omega\left(2^{-k}\right) \leq \int_{n_{0}}^{n} 2^{2 s} \omega\left(2^{-s}\right) d s \leq\left(\frac{4}{\ln 2}\right) \int_{t}^{1} \frac{\omega(z)}{z^{3}} d z .
$$

Collecting the above estimates and using (13), we obtain the desired inequality (24).

From Theorem 2 we derive the following

Corollary 2. If the assumptions of Theorem 2 are satisfied and $\omega(t) \leq M t^{\alpha}, t>0$, with some $m>0$ and $0<\alpha \leq 2$, then

$$
\omega_{2}\left(f, L^{p, q} ; t\right)=\left\{\begin{array}{ll}
O\left(t^{\alpha}\right) & \text { if } 0<\alpha<2, \\
O\left(t^{2}|\ln t|\right) & \text { if } \alpha=2,
\end{array} \text { as } t \rightarrow 0_{+} .\right.
$$

3.3. Now we shall give an analogue of Theorem 1 in the Hölder norm. 
Theorem 3. Suppose that $\omega, \mu \in \Omega^{2}$ and the function $\varphi$ defined by (12) is increasing. If $f \in L^{p, q, \omega}$ with some $1 \leq p \leq \infty$ and $q>0$ and if $r_{0}$ is given by (14), then there exists a positive constant $M$ depending only on $p, q, r_{0}, \mu$ such that

$$
\left\|P_{r}(f ; \cdot)-f(\cdot)\right\|_{p, q, \mu} \leq M\|f\|_{p, q, \omega}^{*} \varphi(r)
$$

for all $r \in\left(0, r_{0}\right] \cap(0,1]$.

Proof: The assumptions on $\omega, \mu$ and $\varphi$ imply that $f, P_{r}(f) \in L^{p, q, \mu}$ if $r \in\left(0, \frac{1}{q}\right)$.

Let $r$ be a fixed point in $\left(0, r_{0}\right] \cap(0,1]$ and let $A=(0, r]$ and $B=(r, 1]$. Then by (9) and (10) we have

$$
\begin{aligned}
\left\|P_{r}(f ; \cdot)\right\|_{p, q, \mu} \leq \| & P_{r}(f ; \cdot)-f(\cdot) \|_{p, q}+\sup _{h \in A} \frac{\left\|\Delta_{h}^{2}\left[P_{r}(f ; \cdot)-f(\cdot)\right]\right\|_{p, q}}{\mu(h)} \\
& +\sup _{h \in B} \frac{\left\|\Delta_{h}^{2}\left[P_{r}(f ; \cdot)-f(\cdot)\right]\right\|_{p, q}}{\mu(h)}:=Y_{1}+Y_{2}+Y_{3} .
\end{aligned}
$$

Using Corollary 1 and by the properties (i), (ii) of functions belonging to $\Omega^{2}$, we get

$$
Y_{1} \leq \frac{5}{2}\left(1-r_{0} q\right)^{-3}\|f\|_{p, q, \omega}^{*} \omega(r) \leq \frac{5}{2}\left(1-r_{0} q\right)^{-3} \mu\left(r_{0}\right)\|f\|_{p, q, \omega}^{*} \varphi(r) .
$$

By (6) and Corollary 1,

$$
Y_{3}=\frac{1}{\mu(r)} \sup _{h \in B} 4 e^{q h}\left\|P_{r}(f ; \cdot)-f(\cdot)\right\|_{p, q} \leq 10 e^{q}\left(1-r_{0} q\right)^{-3}\|f\|_{p, q, \omega}^{*} \varphi(r) .
$$

In view of (5), (17) and (15) we have

$$
\begin{aligned}
Y_{2} & \leq \sup _{h \in A} \frac{\left\|\Delta_{h}^{2} P_{r}(f ; \cdot)\right\|_{p, q}+\left\|\Delta_{h}^{2} f(\cdot)\right\|_{p, q}}{\mu(h)} \leq \sup _{h \in A} \frac{\left\|P_{r}\left(\Delta_{h}^{2} f ; \cdot\right)\right\|_{p, q}+\left\|\Delta_{h}^{2} f(\cdot)\right\|_{p, q}}{\mu(h)} \\
& \leq\left(\frac{1}{1-r_{0} q}+1\right) \sup _{h \in A} \varphi(h) \frac{\left\|\Delta_{h}^{2} f\right\|_{p, q}}{\omega(h)} \leq\left(\frac{1}{1-r_{0} q}+1\right) \varphi(r)\|f\|_{p, q, \omega}^{*} .
\end{aligned}
$$

Summarizing, we obtain (26).

From Theorem 3 follows

Corollary 3. Let $\omega(t)=t^{\alpha}, \mu(t)=t^{\beta}$ for $t>0$ and let $0<\beta<\alpha \leq$ 2. If $f \in L^{p, q, \omega}$ with some $1 \leq p \leq \infty$ and $q>0$, then

$$
\left\|P_{r}(f ; \cdot)-f(\cdot)\right\|_{p, q, \mu}=O\left(r^{\alpha-\beta}\right) \text { as } r \rightarrow 0_{+} .
$$




\section{References}

1. P. L. Butzer and R. J. Nessel, "Fourier Analysis and Approximation," I, II, Birkhäuser, Basel and Academic Press, New York, 1971.

2. M. Górzeńska And L. Rempulska, On the limit properties of the Picard singular integral, Publications de l'Institut Mathematique 55(69) (1994), 39-46.

3. I. I. Ibragimov, "Theory of Approximation by Entire Functions," in Russian, Baku, 1979.

4. R. N. Mohapatra and R. S. Rodríguez, On the rate convergence of singular integrals for Hölder continuous functions, Math. Nachr. 149 (1990), 117-124.

5. J. Prestin ANd S. Prössdorf, Error estimates in generalized trigonometric Hölder-Zygmund norms, Z. Anal. Anwend. 9(4) (1990), 343-349.

6. A. F. Timan, "Theory of Approximation of Functions of a Real Variable," in Russian, Moscow, 1960.

Keywords. Picard singular integral, approximation theorem, Hölder space.

1991 Mathematics subject classifications: 41A25

Institute of Mathematics

Poznań University of Technology

Piotrowo 3A

60-965 Poznań

POLAND

Primera versió rebuda el 2 de Juny de 1995, darrera versió rebuda el 10 de Gener de 1996 